\title{
Some generalizations on the univalence of an integral operator and quasiconformal extensions
}

\author{
Murat Çăglar and Halit Orhan
}




\title{
SOME GENERALIZATIONS ON THE UNIVALENCE OF AN INTEGRAL OPERATOR AND QUASICONFORMAL EXTENSIONS
}

\author{
MURAT ÇAĞLAR AND HALIT ORHAN \\ Received 10 May, 2012
}

\begin{abstract}
By using the method of Loewner chains, we establish some sufficient conditions for the analyticity and univalency of functions defined by an integral operator. Also, we refine the result to a quasiconformal extension criterion with the help of Beckers's method.
\end{abstract}

2000 Mathematics Subject Classification: 30C45; 30C55

Keywords: Loewner chain, univalence criterion, integral operator, quasiconformal extension

\section{INTRODUCTION}

Let $\mathcal{A}$ the class of functions $f$ which are analytic in the open unit disk $U=$ $\{z \in \mathbb{C}:|z|<1\}$ with $f(0)=f^{\prime}(0)-1=0$. We denote by $\mathcal{U}_{r}$ the open disk $\{z \in \mathbb{C}:|z|<r\}$, where $0<r \leq 1$, by $U=U_{1}$ the open unit disk of the complex plane and by $I$ the interval $[0, \infty)$.

Let $k$ be constant in $[0,1)$. Then a homeomorphism $f$ of $G \subset \mathbb{C}$ is said to be $k$-quasiconformal, if $\partial_{z} f$ and $\partial_{\bar{z}} f$ in the distributional sense are locally integrable on $G$ and fulfill the inequality $\left|\partial_{\bar{z}} f\right| \leq k\left|\partial_{z} f\right|$ almost everywhere in $G$. If we do not need to specify $k$, we will simply call $f$ quasiconformal.

Three of the most important and known univalence criteria for analytic functions defined in the open unit disk were obtained by Nehari [14], Ozaki-Nunokawa [17] and Becker [3]. Some extensions of these three criteria were given by [15, 16, 21-25]. Furthermore a lot of univalence criteria have been obtained by different authors (see also [7-9]).

In the present investigation, we will obtain a number of new criteria for the functions defined by the integral operator $\mathcal{F}_{\beta}(z)$. Also, we obtain a refinement to a quasiconformal extension criterion of the main result.

\section{PRELIMinaries}

Before proving our main theorem we present a brief summary of the method of Loewner chains and quasiconformal extension criterion. 
A function $\mathscr{L}(z, t): \mathcal{U} \times[0, \infty) \rightarrow \mathbb{C}$ is said to be subordination chain (or Loewner chain) if:

(i) $\mathscr{L}(z, t)$ is analytic and univalent in $\mathcal{U}$ for all $t \geq 0$.

(ii) $\mathscr{L}(z, t) \prec \mathscr{L}(z, s)$ for all $0 \leq t \leq s<\infty$, where the symbol " $\prec$ " stands for subordination.

To prove our results, we will need the following theorem due to $\mathrm{Ch}$. Pommerenke [20].

Theorem 1. Let $\mathscr{L}(z, t)=a_{1}(t) z+a_{2}(t) z^{2}+\ldots, a_{1}(t) \neq 0$ be analytic in $u_{r}$ for all $t \in I$, locally absolutely continuous in $I$, and locally uniform with respect to $U_{r}$. For almost all $t \in I$, suppose that

$$
z \frac{\partial \mathscr{L}(z, t)}{\partial z}=p(z, t) \frac{\partial \mathscr{L}(z, t)}{\partial t}, \forall z \in \mathcal{U}_{r}
$$

where $p(z, t)$ is analytic in $U$ and satisfies the condition $\Re p(z, t)>0$ for all $z \in$ $\mathcal{U}, t \in I$. If $\left|a_{1}(t)\right| \rightarrow \infty$ for $t \rightarrow \infty$ and $\left\{\mathscr{L}(z, t) / a_{1}(t)\right\}$ forms a normal family in $U_{r}$, then for each $t \in I$, the function $\mathscr{L}(z, t)$ has an analytic and univalent extension to the whole disk $U$.

The method of constructing quasiconformal extension criteria is based on the following result of Becker (see [3], [4] and also [5]).

Theorem 2. Suppose that $\mathscr{L}(z, t)$ is a Loewner chain for which the function $p(z, t)$ given in (2.1) satisfies the condition

$$
\begin{aligned}
p(z, t) & \in \mathcal{U}(k):=\left\{w \in \mathbb{C}:\left|\frac{w-1}{w+1}\right| \leq k\right\} \\
& =\left\{w \in \mathbb{C}:\left|w-\frac{1+k^{2}}{1-k^{2}}\right| \leq \frac{2 k}{1-k^{2}}\right\}, \quad(0 \leq k<1)
\end{aligned}
$$

for all $z \in \mathcal{U}$ and $t \geq 0$. Then $\mathscr{L}(z, t)$ admits a continuous extension to $\bar{U}$ for each $t \geq 0$ and the function $F(z, \bar{z})$ defined by

$$
F(z, \bar{z})=\left\{\begin{array}{cc}
\mathscr{L}(z, 0), & \text { if }|z|<1 \\
\mathscr{L}\left(\frac{z}{|z|}, \log |z|\right), & \text { if }|z| \geq 1
\end{array}\right.
$$

is a $k$-quasiconformal extension of $\mathscr{L}(z, 0)$ to $\mathbb{C}$.

Examples of quasiconformal extension criteria can be found in [1], [2], [6], [13], [19] and more recently in [10-12].

\section{MAin Results}

In this section, using Theorem 1, we obtain certain sufficient conditions for the univalence of an integral operator. 
Theorem 3. Let $m$ be a positive real number and let $\alpha, \beta$ be complex numbers such that $\Re \alpha<1 / 2, \Re \beta>0$ and $f \in \mathcal{A}$. Let $g$ and $h$ be two analytic functions in $u, g(z)=1+b_{1} z+\ldots, h(z)=c_{0}+c_{1} z+\ldots$. If the following inequalities

$$
\left|\frac{f^{\prime}(z)}{g(z)-\alpha}-\frac{m-1}{2}\right|<\frac{m+1}{2}
$$

and

$$
\begin{gathered}
\left.\left|\left(\frac{f^{\prime}(z)}{g(z)-\alpha}-1\right)\right| z\right|^{\beta(m+1)} \\
+\left(1-|z|^{\beta(m+1)}\right)\left[2 z^{\beta} \frac{f^{\prime}(z) h(z)}{g(z)-\alpha}+\frac{1}{\beta} \frac{z g^{\prime}(z)}{g(z)-\alpha}\right] \\
+\frac{z^{\beta+1}\left(1-|z|^{\beta(m+1)}\right)^{2}}{|z|^{\beta(m+1)}}\left[\frac{z^{\beta-1} f^{\prime}(z) h^{2}(z)}{g(z)-\alpha}+\frac{1}{\beta}\left(\frac{g^{\prime}(z) h(z)}{g(z)-\alpha}-h^{\prime}(z)\right)\right]-\frac{m-1}{2} \mid \\
\leq \frac{m+1}{2}
\end{gathered}
$$

are true for all $z \in U$, then the function $\mathcal{F}_{\beta}(z)$ defined by

$$
\mathcal{F}_{\beta}(z)=\left[\beta \int_{0}^{z} u^{\beta-1} f^{\prime}(u) d u\right]^{1 / \beta}
$$

is analytic and univalent in $\mathcal{U}$, where the principal branch is intended.

Proof. We shall prove that there exists a real number $r, r \in(0,1]$ such that the function $\mathscr{L}: U_{r} \times I \rightarrow \mathbb{C}$, defined formally by

$$
\mathscr{L}(z, t)=\left[\beta \int_{0}^{e^{-t} z} u^{\beta-1} f^{\prime}(u) d u+\frac{\left(e^{\beta m t}-e^{-\beta t}\right) z^{\beta}\left(g\left(e^{-t} z\right)-\alpha\right)}{1+\left(e^{\beta m t}-e^{-\beta t}\right) z^{\beta} h\left(e^{-t} z\right)}\right]^{1 / \beta}
$$

is analytic in $U_{r}$ for all $t \in I$.

Because $f \in \mathcal{A}$ we have

$$
f(z)=z+a_{2} z^{2}+\ldots+a_{n} z^{n}+\ldots, \quad \forall z \in \mathcal{U} .
$$

Let us denote by

$$
\varphi_{1}(z, t)=\beta \int_{0}^{e^{-t} z} u^{\beta-1} f^{\prime}(u) d u
$$

We obtain $\varphi_{1}(z, t)=\left(e^{-t} z\right)^{\beta}+\frac{2 \beta a_{2}}{\beta+1}\left(e^{-t} z\right)^{\beta+1}+\ldots$ and we observe that

$$
\varphi_{1}(z, t)=z^{\beta} \varphi_{2}(z, t)
$$


where

$$
\varphi_{2}(z, t)=e^{-\beta t}+\sum_{n=2}^{\infty} \frac{n \beta}{n+\beta-1} a_{n} e^{-(n+\beta-1) t} z^{n-1} .
$$

The function $\varphi_{2}$ is analytic in $\mathcal{U}$ for all $t \in I$, since

$$
\varlimsup_{n \rightarrow \infty} \sqrt[n]{\left|\frac{n \beta}{n+\beta-1} a_{n} e^{-(n+\beta-1) t}\right|}=e^{-t} \varlimsup_{n \rightarrow \infty} \sqrt[n]{\left|a_{n}\right|} .
$$

It is clear that if $z \in \mathcal{U}$, then $e^{-t} z \in \mathcal{U}$ for all $t \in I$ and because $f^{\prime}(0)=1$, there exists a disk $U_{r_{1}}, 0<r_{1} \leq 1$ in which $f^{\prime}\left(e^{-t} z\right) \neq 0$ for all $t \geq 0$.

From the analyticity of $f$ it follows that the function $\varphi_{3}$ is also analytic in $\mathcal{U}_{r_{1}}$, where

$$
\varphi_{3}(z, t)=1+\left(e^{\beta m t}-e^{-\beta t}\right) z^{\beta} h\left(e^{-t} z\right) .
$$

We have $\varphi_{3}(0, t)=1$ and then there exists a disk $U_{r_{2}}, 0<r_{2} \leq r_{1}$ in which $\varphi_{3}(z, t) \neq$ 0 for all $t \geq 0$.

Then the function

$$
\varphi_{4}(z, t)=\varphi_{2}(z, t)+\left(e^{\beta m t}-e^{-\beta t}\right) \frac{\left(g\left(e^{-t} z\right)-\alpha\right)}{\varphi_{3}(z, t)}
$$

is also analytic in $\mathcal{U}_{r_{2}}$ and $\varphi_{4}(0, t)=(1-\alpha) e^{\beta m t}+\alpha e^{-\beta t}$. From $\Re \alpha<1 / 2, \Re \beta>0$ we deduce that $\varphi_{4}(0, t) \neq 0$ for all $t \in I$. Therefore, there exists a disk $\mathcal{U}_{r}, 0<$ $r \leq r_{2}$ in which $\varphi_{4}(0, t) \neq 0$ for all $t \in I$ and we can choose an analytic branch of $\left[\varphi_{4}(z, t)\right]^{1 / \beta}$, denoted by $\varphi_{5}(z, t)$. We choose the uniform branch which is equal to $a_{1}(t)=\left[(1-\alpha) e^{\beta m t}+\alpha e^{-\beta t}\right]^{1 / \beta}$ at the origin, and for $a_{1}(t)$ we get $\lim _{t \rightarrow \infty}\left|a_{1}(t)\right|=$ $\infty$. Moreover, we have $a_{1}(t) \neq 0$ for all $t \geq 0$.

From (3.4)-(3.9) it follows that the relation (3.4) can be written as

$$
\mathscr{L}(z, t)=z \varphi_{5}(z, t)
$$

and hence we obtain that the function $\mathscr{L}(z, t)$ is analytic in $\mathcal{U}_{r}$,

$$
\mathscr{L}(z, t)=a_{1}(t) z+\ldots, \forall z \in U_{r}, \forall t \in I .
$$

$\mathscr{L}(z, t)$ is an analytic function in $\mathcal{U}_{r}$ for all $t \in I$ and then it follows that there is a number $r_{3}, 0<r_{3}<r$ and a positive constant $K=K\left(r_{3}\right)$ such that

$$
\left|\frac{\mathscr{L}(z, t)}{a_{1}(t)}\right|<K, \forall z \in U_{r_{3}}, t \geq 0 .
$$

Then, by Montel's theorem, it follows that $\left\{\frac{\mathscr{L}(z, t)}{a_{1}(t)}\right\}_{t \geq 0}$ is a normal family in $U_{r_{3}}$.

From (3.10) we have

$$
\frac{\partial \mathscr{L}(z, t)}{\partial t}=z \frac{\partial \varphi_{5}(z, t)}{\partial t} .
$$


It is clear that $\frac{\partial \varphi_{5}(z, t)}{\partial t}$ is an analytic function in $U_{r_{3}}$ and then $\frac{\partial \mathscr{L}(z, t)}{\partial t}$ is also an analytic function in $U_{r_{3}}$. Then, for all fixed numbers $T>0$ and $r_{4}, 0<r_{4}<r_{3}$, there exists a constant $K_{1}>0$ (which depends on $T$ and $r_{4}$ ) such that

$$
\left|\frac{\partial \mathscr{L}(z, t)}{\partial t}\right|<K_{1}, \forall z \in U_{r_{4}} \text { and } t \in[0, T] .
$$

Therefore, the function $\mathscr{L}(z, t)$ is locally absolutely continuous in $[0, \infty)$ and is locally uniform with respect to $\mathcal{U}_{r_{4}}$.

Since $\frac{\partial \mathscr{L}(z, t)}{\partial t}$ is analytic in $\mathcal{U}_{r_{4}}$, from (3.11) it follows that there is a number $r_{0}$, $0<r_{0}<r_{4}$, such that $\frac{1}{z} \frac{\partial \mathscr{L}(z, t)}{\partial t} \neq 0, \forall z \in \mathcal{U}_{r_{0}}$, so the function

$$
p(z, t)=z \frac{\partial \mathscr{L}(z, t)}{\partial z} / \frac{\partial \mathscr{L}(z, t)}{\partial t}
$$

is analytic in $U_{r_{0}}$ for all $t \geq 0$.

In order to prove that the function $p(z, t)$ has an analytic extension with positive real part in $U$ for all $t \geq 0$, it is sufficient to prove that the function $w(z, t)$ defined in $u_{r_{0}}$ by

$$
w(z, t)=\frac{p(z, t)-1}{p(z, t)+1}
$$

can be extended analytically in $U,|w(z, t)|<1$ for all $z \in \mathcal{U}$ and $t \geq 0$.

After some calculations we obtain:

$$
w(z, t)=\frac{2}{m+1} \mathcal{E}(z, t)-\frac{m-1}{m+1},
$$

where

$$
\begin{aligned}
\mathcal{E}(z, t)= & e^{-\beta(m+1) t}\left(\frac{f^{\prime}\left(e^{-t} z\right)}{g\left(e^{-t} z\right)-\alpha}-1\right) \\
& +\left(1-e^{-\beta(m+1) t}\right)\left[2 e^{-\beta t} z^{\beta} \frac{f^{\prime}\left(e^{-t} z\right) h\left(e^{-t} z\right)}{g\left(e^{-t} z\right)-\alpha}+\frac{e^{-t} z}{\beta} \frac{g^{\prime}\left(e^{-t} z\right)}{g\left(e^{-t} z\right)-\alpha}\right] \\
& +\frac{e^{-\beta t} z^{\beta}\left(1-e^{-\beta(m+1) t}\right)^{2}}{e^{-\beta(m+1) t}} \\
& \times\left[e^{-\beta t} z^{\beta} \frac{f^{\prime}\left(e^{-t} z\right) h^{2}\left(e^{-t} z\right)}{g\left(e^{-t} z\right)-\alpha}\right. \\
& \left.+\frac{e^{-t} z}{\beta}\left(\frac{h\left(e^{-t} z\right) g^{\prime}\left(e^{-t} z\right)}{g\left(e^{-t} z\right)-\alpha}-h^{\prime}\left(e^{-t} z\right)\right)\right]
\end{aligned}
$$

for $z \in \mathcal{U}$ and $t \geq 0$. 
The inequality $|w(z, t)|<1$ for all $z \in \mathcal{U}$ and $t \geq 0$, where $w(z, t)$ defined by (3.12), is equivalent to

$$
\left|\mathcal{E}(z, t)-\frac{m-1}{2}\right|<\frac{m+1}{2}, \forall z \in \mathcal{U} \text { and } t \geq 0 .
$$

Define

$$
\mathscr{H}(z, t)=\mathscr{E}(z, t)-\frac{m-1}{2}, \forall z \in \mathcal{U} \text { and } t \geq 0 .
$$

In view of (3.1) and (3.2), from (3.13) and (3.15) we have

$$
|\mathscr{H}(z, 0)|=\left|\left(\frac{f^{\prime}(z)}{g(z)-\alpha}-1\right)-\frac{m-1}{2}\right|<\frac{m+1}{2} .
$$

Let $t>0, z \in \mathcal{U}-\{0\}$. In this case the function $\mathscr{H}(z, t)$ is analytic in $\bar{U}$ because $\left|e^{-t} z\right| \leq e^{-t}<1$, for all $z \in \bar{U}$. Using the maximum principle for $z \in \mathcal{U}$ and $t>0$ we have

$$
|\mathcal{H}(z, t)|<\max _{|\xi|=1}|\mathcal{H}(\xi, t)|=\left|\mathscr{H}\left(e^{i \theta}, t\right)\right|,
$$

where $\theta=\theta(t)$ is a real number.

Let $u=e^{-t} e^{i \theta}$. We have $|u|=e^{-t}$ and $e^{-\beta(m+1) t}=\left(e^{-t}\right)^{\beta(m+1)}=|u|^{\beta(m+1)}$. From (3.13), we have

$$
\begin{aligned}
\left|\mathcal{E}\left(e^{i \theta}, t\right)\right|= & \left.|| u\right|^{\beta(m+1)}\left(\frac{f^{\prime}(u)}{g(u)-\alpha}-1\right) \\
& +\left(1-|u|^{\beta(m+1)}\right)\left[\frac{2 u^{\beta} f^{\prime}(u) h(u)}{g(u)-\alpha}+\frac{u}{\beta} \frac{g^{\prime}(u)}{g(u)-\alpha}\right] \\
& +\frac{u^{\beta}\left(1-|u|^{\beta(m+1)}\right)^{2}}{|u|^{\beta(m+1)}} \\
& \times\left[\frac{u^{\beta} f^{\prime}(u) h^{2}(u)}{g(u)-\alpha}+\frac{u}{\beta}\left(\frac{h(u) g^{\prime}(u)}{g(u)-\alpha}-h^{\prime}(u)\right)\right]-\frac{m-1}{2} \mid .
\end{aligned}
$$

Since $u \in U$, the inequality (3.2) implies that

$$
\left|\mathcal{H}\left(e^{i \theta}, t\right)\right| \leq \frac{m+1}{2},
$$

and from (3.16) and (3.17) it follows that the inequality (3.14)

$$
|\mathscr{H}(z, t)|=\left|\mathcal{S}(z, t)-\frac{m-1}{2}\right|<\frac{m+1}{2}
$$

is satisfied for all $z \in U$ and $t \in I$. Therefore $|w(z, t)|<1$, for all $z \in U$ and $t \geq 0$.

Since all the conditions of Theorem 1 are satisfied, we obtain that the function $\mathscr{L}(z, t)$ has an analytic and univalent extension to the whole unit disk $\mathcal{U}$, for all $t \in I$. 
For $t=0$ we have $\mathscr{L}(z, 0)=\mathscr{F}_{\beta}(z)$, for $z \in U$ and therefore, the function $\mathscr{F}_{\beta}(z)$ is analytic and univalent in $U$.

For $g=f^{\prime}$ in Theorem 3, we obtain another univalence criterion as follows.

Corollary 1. Let $m$ be a positive real number and let $\alpha, \beta$ be complex numbers such that $\Re \alpha<1 / 2, \Re \beta>0$ and $f \in \mathcal{A}$. Let $h$ be an analytic functions in $U, h(z)=$ $c_{0}+c_{1} z+\ldots$. If the following inequalities

$$
\left|\frac{f^{\prime}(z)}{f^{\prime}(z)-\alpha}-\frac{m+1}{2}\right|<\frac{m+1}{2}
$$

and

$$
\begin{gathered}
\left.\left|\left(\frac{f^{\prime}(z)}{f^{\prime}(z)-\alpha}-1\right)\right| z\right|^{\beta(m+1)} \\
+\left(1-|z|^{\beta(m+1)}\right)\left[2 z^{\beta} \frac{f^{\prime}(z) h(z)}{f^{\prime}(z)-\alpha}+\frac{1}{\beta} \frac{z f^{\prime \prime}(z)}{f^{\prime}(z)-\alpha}\right] \\
+\frac{z^{\beta+1}\left(1-|z|^{\beta(m+1)}\right)^{2}}{|z|^{\beta(m+1)}}\left[\frac{z^{\beta-1} f^{\prime}(z) h^{2}(z)}{f^{\prime}(z)-\alpha}+\frac{1}{\beta}\left(\frac{f^{\prime \prime}(z) h(z)}{f^{\prime}(z)-\alpha}-h^{\prime}(z)\right)\right] \\
-\frac{m-1}{2} \mid \\
\leq \frac{m+1}{2}
\end{gathered}
$$

are true for all $z \in \mathcal{U}$, then the function $\mathcal{F}_{\beta}(z)$ defined by (3.3) is analytic and univalent in $\mathcal{U}$, where the principal branch is intended.

If we choose $h=f^{\prime \prime}$ in Corollary 1, we have another univalence criterion as follows.

Corollary 2. Let $m$ be a positive real number and let $\alpha, \beta$ be complex numbers such that $\Re \alpha<1 / 2, \Re \beta>0$ and $f \in \mathcal{A}$. Let $h$ be an analytic functions in $U, h(z)=$ $c_{0}+c_{1} z+\ldots$. If the following inequalities

$$
\left|\frac{f^{\prime}(z)}{f^{\prime}(z)-\alpha}-\frac{m+1}{2}\right|<\frac{m+1}{2},
$$

and

$$
\begin{gathered}
\left.\left|\left(\frac{f^{\prime}(z)}{f^{\prime}(z)-\alpha}-1\right)\right| z\right|^{\beta(m+1)} \\
+\left(1-|z|^{\beta(m+1)}\right)\left[2 z^{\beta} \frac{f^{\prime}(z) h(z)}{f^{\prime}(z)-\alpha}+\frac{1}{\beta} \frac{z f^{\prime \prime}(z)}{f^{\prime}(z)-\alpha}\right]
\end{gathered}
$$




$$
\begin{gathered}
+\frac{z^{\beta+1}\left(1-|z|^{\beta(m+1)}\right)^{2}}{|z|^{\beta(m+1)}}\left[\frac{z^{\beta-1} f^{\prime}(z) h^{2}(z)}{f^{\prime}(z)-\alpha}+\frac{1}{\beta}\left(\frac{f^{\prime \prime}(z) h(z)}{f^{\prime}(z)-\alpha}-h^{\prime}(z)\right)\right] \\
-\frac{m-1}{2} \mid \\
\leq \frac{m+1}{2}
\end{gathered}
$$

are true for all $z \in U$, then the function $\mathcal{F}_{\beta}(z)$ defined by (3.3) is analytic and univalent in $\mathcal{U}$, where the principal branch is intended.

Corollary 3. Let $m$ be a positive real number and let $\alpha, \beta$ be complex numbers such that $\Re \alpha<1 / 2, \Re \beta>0$ and $f \in \mathcal{A}$. If the following inequalities

$$
\left|\frac{f^{\prime}(z)}{f^{\prime}(z)-\alpha}-\frac{m+1}{2}\right|<\frac{m+1}{2}
$$

and

$$
\begin{aligned}
& \left.\left|\left(\frac{f^{\prime}(z)}{f^{\prime}(z)-\alpha}-1\right)\right| z\right|^{\beta(m+1)}+\left(1-|z|^{\beta(m+1)}\right)\left[\frac{1}{\beta} \frac{z f^{\prime \prime}(z)}{f^{\prime}(z)-\alpha}\right]-\frac{m-1}{2} \mid \\
& \leq \frac{m+1}{2}
\end{aligned}
$$

are true for all $z \in \mathcal{U}$, then the function $\mathcal{F}_{\beta}(z)$ defined by (3.3) is analytic and univalent in $\mathcal{U}$, where the principal branch is intended.

Proof. It results from Corollary 1 with $g=f^{\prime}$ and $h=0$.

If we consider $g(z)=f^{\prime}, h(z)=-\frac{1}{2} \frac{f^{\prime \prime}}{f^{\prime}}, \alpha=0, \beta=1$ in Theorem 3, we obtain another univalence criterion as follows.

Corollary 4. Let $m$ be a positive real number and $f \in \mathcal{A}$. If the following inequality

$$
\left|\frac{z^{2}\left(1-|z|^{m+1}\right)^{2}}{|z|^{m+1}}\left(\frac{1}{2}\{f ; z\}\right)-\frac{m-1}{2}\right| \leq \frac{m+1}{2}
$$

where

$$
\{f ; z\}=\left(\frac{f^{\prime \prime}(z)}{f^{\prime}(z)}\right)^{\prime}-\frac{1}{2}\left(\frac{f^{\prime \prime}(z)}{f^{\prime}(z)}\right)^{2}
$$

is true for all $z \in \mathcal{U}$, then the function $f(z)$ is analytic and univalent in $\mathcal{U}$, where the principal branch is intended.

Setting $\alpha=0$ in Corollary 3 we have another univalence criterion as follows. 
Corollary 5. Let $m$ be a positive real number and let $\beta$ be complex number such that $\Re \beta>0$ and $f \in \mathcal{A}$. If the following inequality

$$
\left|\frac{\left(1-|z|^{\beta(m+1)}\right)}{\beta}\left(\frac{z f^{\prime \prime}(z)}{f^{\prime}(z)}\right)-\frac{m-1}{2}\right| \leq \frac{m+1}{2}
$$

is true for all $z \in \mathcal{U}$, then the function $\mathcal{F}_{\beta}(z)$ defined by (3.3) is analytic and univalent in $U$, where the principal branch is intended.

Corollary 6. Let $m$ be a positive real number and let $\beta$ be complex number with $\Re \beta>0$ and $f \in \mathcal{A}$. If the following inequality

$$
\left|\frac{\left(1-|z|^{(m+1) \Re \beta}\right)}{\Re \beta}\left(\frac{z f^{\prime \prime}(z)}{f^{\prime}(z)}\right)\right| \leq 1
$$

is true for all $z \in U$, then the function $\mathcal{F}_{\beta}(z)$ defined by (3.3) is analytic and univalent in $U$, where the principal branch is intended.

Proof. It can be proved (see [18]) that for $z \in \mathcal{U} \backslash\{0\}, \Re \beta>0$ and $m \in \mathbb{R}_{+}$

$$
\left|\frac{1-|z|^{(m+1) \beta}}{\beta}\right| \leq \frac{1-|z|^{(m+1) \Re \beta}}{\Re \beta} .
$$

For $m \geq 1$, we have

$$
\begin{gathered}
\left|\frac{1-|z|^{(m+1) \beta}}{\beta}\left(\frac{z f^{\prime \prime}(z)}{f^{\prime}(z)}\right)-\frac{m-1}{2}\right| \\
\leq\left|\frac{1-|z|^{(m+1) \beta}}{\beta}\left(\frac{z f^{\prime \prime}(z)}{f^{\prime}(z)}\right)\right|+\frac{m-1}{2} \\
\leq \frac{1-|z|^{(m+1) \Re \beta}}{\Re \beta}\left|\frac{z f^{\prime \prime}(z)}{f^{\prime}(z)}\right|+\frac{m-1}{2} \\
\leq 1+\frac{m-1}{2}=\frac{m+1}{2} .
\end{gathered}
$$

Since inequalities (3.1) and (3.2) are satisfied, making use of Theorem 3, we can conclude that the function $\mathcal{F}_{\beta}$ is analytic and univalent in $\mathcal{U}$.

Putting $g(z)=\left(\frac{f(z)}{z}\right)^{2}, h(z)=0, \alpha=0$, in Theorem 3, we get the univalence criterion as follows.

Corollary 7. Let $m$ be a positive real number and let $\beta$ be complex number such that $\Re \beta>0$ and $f \in \mathcal{A}$. If the following inequalities

$$
\left|\frac{z^{2} f^{\prime}(z)}{f^{2}(z)}-\frac{m+1}{2}\right|<\frac{m+1}{2}
$$


and

$$
\begin{aligned}
& \left.\left|\left(\frac{z^{2} f^{\prime}(z)}{f^{2}(z)}-1\right)\right| z\right|^{\beta(m+1)}+\frac{2\left(1-|z|^{\beta(m+1)}\right)}{\beta}\left(\frac{z f^{\prime}(z)}{f(z)}-1\right)-\frac{m-1}{2} \mid \\
& \leq \frac{m+1}{2}
\end{aligned}
$$

are true for all $z \in \mathcal{U}$, then the function $\mathcal{F}_{\beta}(z)$ defined by (3.3) is analytic and univalent in $\mathcal{U}$, where the principal branch is intended.

Corollary 8. Let $m$ be a positive real number and $f \in \mathcal{A}$. If the following inequality

$$
\begin{aligned}
& \mid z\left(1-|z|^{m+1}\right)\left(2 f^{\prime \prime}(z)+\frac{f^{\prime \prime}(z)}{f^{\prime}(z)}\right) \\
& +\frac{z^{2}\left(1-|z|^{m+1}\right)^{2}}{|z|^{m+1}}\left(\frac{\left(f^{\prime \prime}(z)\right)^{2}}{f^{\prime}(z)}+\left(f^{\prime \prime}(z)\right)^{2}-f^{\prime \prime \prime}(z)\right)-\frac{m-1}{2} \mid \\
& \leq \frac{m+1}{2}
\end{aligned}
$$

is true for all $z \in \mathcal{U}$, then the function $f(z)$ is analytic and univalent in $\mathcal{U}$, where the principal branch is intended.

Proof. It results from Corollary 2 with $\alpha=0, \beta=1$.

Remark 1. (1) Putting $g(z)=f^{\prime}(z), h(z)=0, \alpha=0, \beta=m=1$ in Theorem 3, we have Becker's criterion [3].

(2) If we consider $g(z)=f^{\prime}(z), h(z)=-\frac{1}{2} \frac{f^{\prime \prime}(z)}{f^{\prime}(z)}, \alpha=0, \beta=m=1$ in Theorem 3 , we obtain the univalence criterion due to Nehari [14].

(3) Setting $g(z)=\left(\frac{f(z)}{z}\right)^{2}, h(z)=\frac{1}{z}-\frac{f(z)}{z^{2}}, \alpha=0, \beta=m=1$ in Theorem 3, we get the univalence criterion due to Ozaki-Nunokawa [17].

(4) For $g(z)=f^{\prime}(z), h(z)=\frac{1}{z}-\frac{f(z)}{f(z)}, \alpha=0, \beta=m=1$ in Theorem 3, we arrive at Goluzin's criterion for univalence [9].

(5) For $m=1$ in Corollary 6, we obtain the univalence criterion due to Pascu [18].

(6) If we consider $g(z)=f^{\prime}(z), h(z)=0, \beta=1$ in Theorem 3, we have results of Raducanu et al. [23].

(7) Putting $\alpha=0, \beta=m=1$ in Theorem 3, we get the univalence criterion due to Ovesea-Tudor and Owa [16].

Example 1. Let the function

$$
f(z)=\frac{z}{1-\frac{z^{2}}{2}}
$$


Then $f$ is univalent in $\mathcal{U}$ and the function

$$
\widetilde{F}_{2}(z)=\left(2 \int_{0}^{z} u f^{\prime}(u) d u\right)^{\frac{1}{2}}
$$

is analytic and univalent in $U$.

Infact, from equality (3.29) for $m=1$, we have

$$
\frac{z^{2} f^{\prime}(z)}{f^{2}(z)}-1=\frac{z^{2}}{2}
$$

It is clear that the condition (3.29) of the Corollary 7 is satisfied for $m=1$, and then the function $f$ is univalent in $\mathcal{U}$.

Taking into account (3.34), the condition (3.30) of Corollary 7 becomes for $\beta=2$, $m=1$,

$$
\begin{aligned}
\left.\left|\frac{z^{2}}{2}\right| z\right|^{4}+\left(1-|z|^{4}\right) \frac{2 z^{2}}{2-z^{2}} \mid & \leq \frac{|z|^{6}}{2}+2\left(1-|z|^{4}\right)|z|^{2} \\
& =\frac{1}{2}\left(4|z|^{2}-3|z|^{6}\right)<1
\end{aligned}
$$

because the greatest value of the function $g(x)=4 x^{2}-3 x^{6}$, for $x \in[0,1]$ is taken for $x=\sqrt{\frac{2}{3}}$ and $g\left(\sqrt{\frac{2}{3}}\right)=\frac{24}{27}$. Therefore the function $\mathscr{F}_{2}(z)$ defined by (3.33) is analytic and univalent in $\mathcal{U}$.

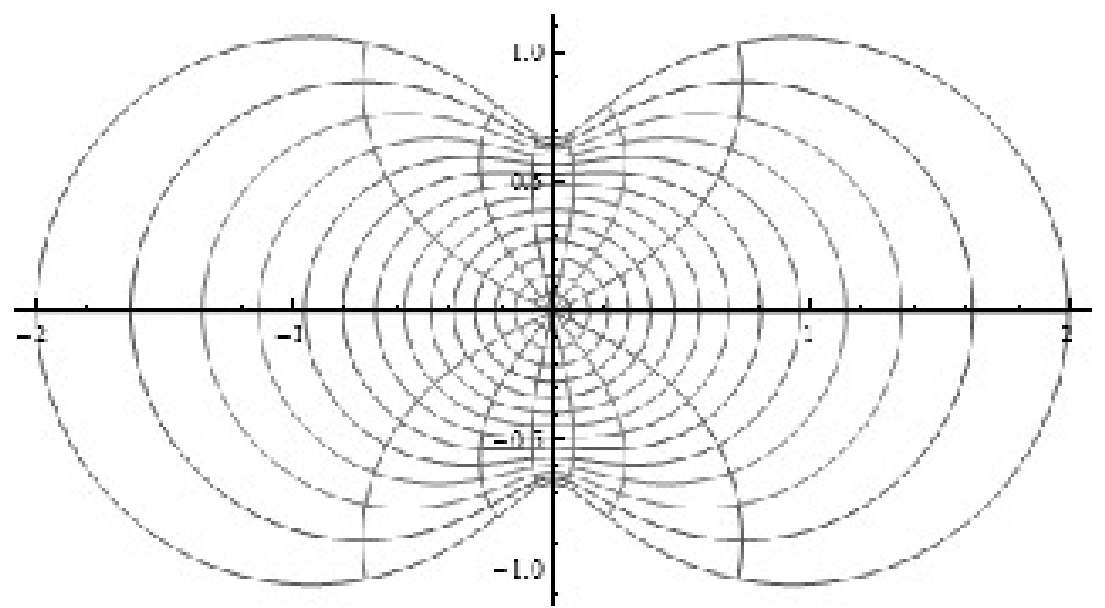

FIGURE 1. $f(z)=\frac{z}{1-\frac{z^{2}}{2}}$ 


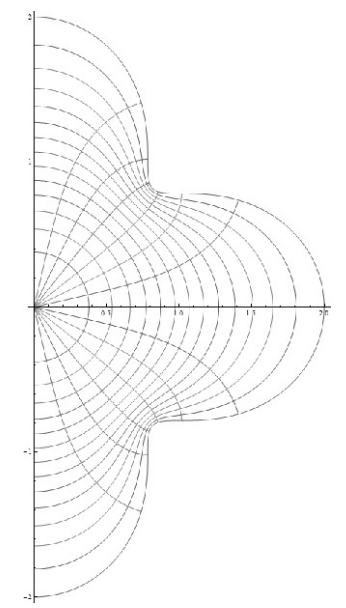

FIGURE 2. $\mathcal{F}_{2}(z)=\left(4 \int_{0}^{z} \frac{2+u^{2}}{\left(2-u^{2}\right)^{2}} d u\right)^{\frac{1}{2}}$

\section{QUASICONFORMAL EXTENSION CRITERION}

In this section we will generalize the univalence condition given in Theorem 3 to a quasiconformal extension criterion.

Theorem 4. Let $m$ be a positive real number and let $\alpha, \beta$ be complex numbers such that $\Re \alpha<1 / 2, \Re \beta>0, f \in \mathcal{A}$ and $k \in[0,1)$. Let $g$ and $h$ be two analytic functions in $U, g(z)=1+b_{1} z+\ldots, h(z)=c_{0}+c_{1} z+\ldots$. If the following inequalities

$$
\left|\frac{f^{\prime}(z)}{g(z)-\alpha}-\frac{m+1}{2}\right|<k \frac{m+1}{2},
$$

and

$$
\begin{gathered}
\left.\left|\left(\frac{f^{\prime}(z)}{g(z)-\alpha}-1\right)\right| z\right|^{\beta(m+1)} \\
+\left(1-|z|^{\beta(m+1)}\right)\left[2 z^{\beta} \frac{f^{\prime}(z) h(z)}{g(z)-\alpha}+\frac{1}{\beta} \frac{z g^{\prime}(z)}{g(z)-\alpha}\right] \\
+\frac{z^{\beta+1}\left(1-|z|^{\beta(m+1)}\right)^{2}}{|z|^{\beta(m+1)}}\left[\frac{z^{\beta-1} f^{\prime}(z) h^{2}(z)}{g(z)-\alpha}+\frac{1}{\beta}\left(\frac{g^{\prime}(z) h(z)}{g(z)-\alpha}-h^{\prime}(z)\right)\right]-\frac{m-1}{2} \mid \\
\leq k \frac{m+1}{2}
\end{gathered}
$$

is true for all $z \in \mathcal{U}$, then the function $\mathcal{F}_{\beta}(z)$ given by (3.3) has a $k$-quasiconformal extension to $\mathbb{C}$. 
Proof. Set

$$
\mathscr{L}(z, t)=\left[\beta \int_{0}^{e^{-t} z} u^{\beta-1} f^{\prime}(u) d u+\frac{\left(e^{\beta m t}-e^{-\beta t}\right) z^{\beta}\left(g\left(e^{-t} z\right)-\alpha\right)}{1+\left(e^{\beta m t}-e^{-\beta t}\right) z^{\beta} h\left(e^{-t} z\right)}\right]^{1 / \beta}
$$

In the proof of Theorem 3 has been shown that the function $\mathscr{L}(z, t)$ given by $(4.3)$ is a subordination chain in $\mathcal{U}$. Then we have

$$
\begin{aligned}
& \left|\frac{p(z, t)-1}{p(z, t)+1}\right|=\mid \frac{2}{m+1}\left\{e^{-\beta(m+1) t}\left(\frac{f^{\prime}\left(e^{-t} z\right)}{g\left(e^{-t} z\right)-\alpha}-1\right)\right. \\
& +\left(1-e^{-\beta(m+1) t}\right)\left[2 e^{-\beta t} z^{\beta} \frac{f^{\prime}\left(e^{-t} z\right) h\left(e^{-t} z\right)}{g\left(e^{-t} z\right)-\alpha}+\frac{e^{-t} z}{\beta} \frac{g^{\prime}\left(e^{-t} z\right)}{g\left(e^{-t} z\right)-\alpha}\right] \\
& +\frac{e^{-\beta t} z^{\beta}\left(1-e^{-\beta(m+1) t}\right)^{2}}{e^{-\beta(m+1) t}} \\
& \left.\times\left[e^{-\beta t} z^{\beta} \frac{f^{\prime}\left(e^{-t} z\right) h^{2}\left(e^{-t} z\right)}{g\left(e^{-t} z\right)-\alpha}+\frac{e^{-t} z}{\beta}\left(\frac{h\left(e^{-t} z\right) g^{\prime}\left(e^{-t} z\right)}{g\left(e^{-t} z\right)-\alpha}-h^{\prime}\left(e^{-t} z\right)\right)\right]\right\} \\
& -\frac{m-1}{m+1} \\
& \leq k \text {. }
\end{aligned}
$$

The right hand of (4.4) always less than or equal to $k$ from (4.2) and therefore $\mathcal{F}_{\beta}$ can be extended to $k$ quasiconformal mapping to $\mathbb{C}$ by Theorem 1 and Theorem 2 .

\section{ACKNOWLEDGEMENT}

The authors thank the referees for their valuable suggestions to improve the paper.

The present investigation was supported by Ataturk University Rectorship under "The Scientific and Research Project of Ataturk University", Project No: 2012/173.

\section{REFERENCES}

[1] L. V. Ahlfors, "Sufficient conditions for quasi-conformal extension," in Discontin. Groups Riemann Surf., Proc. 1973 Conf. Univ. Maryland, 1974, pp. 23-29.

[2] J. Anderson and A. Hinkkanen, "Univalence criteria and quasiconformal extensions," Trans. Am. Math. Soc., vol. 324, no. 2, pp. 823-842, 1991.

[3] J. Becker, "Löwnersche differentialgleichung und quasikonform fortsetzbare schlichte funktionen," J. Reine Angew. Math., vol. 255, pp. 23-43, 1972.

[4] J. Becker, "über die lösungsstruktur einer differentialgleichung in der konformen abbildung," $J$. Reine Angew. Math., vol. 285, pp. 66-74, 1976.

[5] J. Becker, "Conformal mappings with quasiconformal extensions," in Aspects of contemporary complex analysis. Durham/Engl.: Proc. instr. Conf., 1980, vol. 1979, pp. 37-77.

[6] T. Betker, "Löwner chains and quasiconformal extensions," Complex Variables, Theory Appl., vol. 20, no. 1-4, pp. 107-111, 1992. 
[7] E. Denız and H. Orhan, "Some notes on extensions of basic univalence criteria," J. Korean Math. Soc., vol. 48, no. 1, pp. 179-189, 2011.

[8] E. Deniz, D. R $\longleftarrow$ aducanu, and H. Orhan, "On an improvement of a univalence criterion," Math. Balk., New Ser., vol. 24, no. 1-2, pp. 33-39, 2010.

[9] G. M. Goluzin, Geometric theory of functions of a complex variable, ser. Translations of Mathematical Monographs. Providence, R. I.: American Mathematical Society (AMS), 1969, vol. 26.

[10] I. Hotta, "Explicit quasiconformal extensions and Löwner chains," Proc. Japan Acad., Ser. A, vol. 85, no. 8, pp. 108-111, 2009.

[11] I. Hotta, "Löewner chains and quasiconformal extension of univalent functions," Ph.D. dissertation, Tohoku Univ., 2010.

[12] I. Hotta, "Löwner chains with complex leading coefficient," Monatsh. Math., vol. 163, no. 3, pp. 315-325, 2011.

[13] J. G. Krzyz, "Convolution and quasiconformal extension," Comment. Math. Helv., vol. 51, pp. 99-104, 1976.

[14] Z. Nehari, "The Schwarzian derivative and schlicht functions," Bull. Am. Math. Soc., vol. 55, pp. 545-551, 1949.

[15] H. Ovesea, "An univalence criterion and the Schwarzian derivative," Novi Sad J. Math., vol. 26, no. 1 , pp. 69-76, 1996.

[16] H. Ovesea-Tudor and S. Owa, "An extension of the univalence criteria of Nehari and Ozaki and Nunokawa," Hokkaido Math. J., vol. 34, no. 3, pp. 533-539, 2005.

[17] S. Ozaki and M. Nunokawa, "The Schwarzian derivative and univalent functions," Proc. Am. Math. Soc., vol. 33, pp. 392-394, 1972.

[18] N. N. Pascu, "An improvement of Becker's univalence criterion," in Proc. Commem. Sess. Simion Stoilow, Brasov/Rom., 1988, vol. 1987, pp. 43-48.

[19] J. A. Pfaltzgraff, " $k$-quasiconformal extension criteria in the disk," Complex Variables, Theory Appl., vol. 21, no. 3-4, pp. 293-301, 1993.

[20] C. Pommerenke, Univalent functions. With a chapter on quadratic differentials by Gerd Jensen, ser. Studia Mathematica/Mathematische Lehrbücher. Göttingen: Vandenhoeck \& Ruprecht, 1975, vol. XXV.

[21] D. Răducanu, "On a univalence criterion," Mathematica, vol. 37(60), no. 1-2, pp. 227-231, 1995.

[22] D. Răducanu, "A univalence criterion for analytic functions in the unit disk," Mathematica, vol. 46(69), no. 2, pp. 213-216, 2004.

[23] D. Răducanu, H. Orhan, and E. Deniz, "On some sufficient conditions for univalence," An. Ştiinţ. Univ. "Ovidius” Constanţa, Ser. Mat., vol. 18, no. 2, pp. 217-222, 2010.

[24] D. Răducanu, I. Radomir, M. E. Gageonea, and N. R. Pascu, "A generalization of OzakiNunokawa's univalence criterion," JIPAM, J. Inequal. Pure Appl. Math., vol. 5, no. 4, p. 4, 2004.

[25] H. Tudor, "New univalence criteria," Stud. Univ. Babeş-Bolyai, Math., vol. 52, no. 2, pp. 127-132, 2007.

\section{Authors' addresses}

\section{Murat Çağlar}

Ataturk University, Faculty of Science, Department of Mathematics, 25240, Erzurum, Turkey

E-mail address: mcaglar25egmail.com

\section{Halit Orhan}

Ataturk University, Faculty of Science, Department of Mathematics, 25240, Erzurum, Turkey

E-mail address: orhanhalit607@gmail.com 